\title{
Can we really continue to diagnose high grade dysplasia in Barrett's esophagus in Europe without magnified virtual chromo-endoscopy?
}

Author

Institution
Mathieu Pioche

Endoscopy Unit, University Hospital Edouard Herriot, Lyon, France
Bibliography

DOI http://dx.doi.org/

10.1055/s-0034-1391363

Published online: 3.2.2015

Endoscopy International Open

2015; 03: E29-E30

(c) Georg Thieme Verlag KG

Stuttgart $\cdot$ New York

E-ISSN 2196-9736

\section{Corresponding author}

Mathieu Pioche, MD

Gastroenterology and Endoscopy

Edouard Herriot Hospital

5 Place d'Arsonval

69437 Lyon Cédex

Fax: +33 472110147

Mathieu.pioche@chu-lyon.fr
License terms

$(\circledast) \Theta \circledast$
In the endoscopic diagnosis of Barrett's esophagus, the recurring preoccupation is to replace Seattle random biopsies with targeted biopsies. In this editorial, we immediately evade the question because even with the last generation of endoscopes, the diagnosis of low grade dysplasia (LGD) remains unsuccessful [1], so that random biopsies currently cannot be avoided. Nevertheless, there is a need for better detection of high grade dysplasia (HGD) and/or superficial carcinoma and for better targeted biopsies before random biopsies are performed.

Standard white light imaging (WLI) endoscopy makes it possible to detect surface abnormalities, such as depressed areas and elevated components, although the dysplastic lesions of Barrett's esophagus are usually flat (Paris classification 0 IIb) [2]. Based on this observation, the examination needs to be more subtle and focused on mucosal and vascular patterns, which reflect the structure of glands and vessels. On pathologic examination, HGD is associated with both cellular and architectural changes of the mucosa, showing irregular crypts and irregular vessels.

Narrow-band imaging (NBI) enhances these patterns [3] and makes it possible to observe some differences between intestinal metaplasia and dysplastic flat areas. NBI quickly appeared to be as effective as colorant dying [4] and more effective than WLI [5] for detecting areas of HGD. NBI demonstrated a high degree of effectiveness in diagnosing these areas when the random biopsy protocol was used as the gold standard [6, 7]. In the multicenter experience of Sharma et al, more dysplastic areas were detected during NBI examination without magnification than during WLI ( $30 \%$ vs $21 \%$, with half as many biopsies [7].

Various classifications have been proposed to assess mucosal and vascular patterns more reliably $[4,8,9]$. Regular mucosal patterns may be long and straight, cerebriform, villous, or atrophic, and they are associated with non-dysplastic mu- cosa. On the contrary, irregular mucosal patterns are rough, distorted, and disrupted or have destroyed villi, and they demonstrate an irregular distribution of features. These mucosal characteristics correspond to dysplastic areas and are frequently seen in HGD; they can be revealed by the combination of NBI and spraying with peracetic acid. Regular vascular patterns may look like a network, mesh, honeycomb, or DNA spiral, as opposed to irregular vascular patterns, in which large or spiral vessels are seen with mixed avascular and crowded areas.

These classification systems suffer from poor to moderate inter-observer concordance [10], mainly because the vascular network is difficult to interpret. Currently, a simplified distinction between regular and irregular patterns is often used, as in the study of Singh et al [11], but this is still controversial because of the relatively low rates of specificity $(72 \%)$, sensitivity $(47 \%)$, and inter-observer concordance $(\mathrm{K}=0.40)$ in the diagnosis of dysplasia (LGD, HGD, and intramucosal carcinoma) when it is used for studies done without magnification [1].

Magnification is theoretically beneficial by allowing a detailed examination of Barrett's mucosa and revealing the subtle architectural changes of areas of HGD. With magnification, the negative predictive value for HGD improves to $98 \%[4,9]$ when regular villous and vascular patterns are recognized in Barrett's esophagus. In the study that appears in this issue of Endoscopy International Open, Singh et al report a new international validation of the simplified classification for diagnosing neoplasia in Barrett's esophagus with NBI and WLI magnified endoscopy. This study confirms the very high negative predictive value (99\%) of magnified endoscopy for the diagnosis of HGD when it is performed by expert endoscopists in Asia-Pacific countries. These endoscopists are skilled in magnified endoscopy because of their large experience in screening for gastric cancer, 
but some of them have a relatively limited dedicated experience in Barrett's esophagus because of the rarity of the disease in Asia. This report underscores the Asian doctors' ability to recognize neoplastic vascular and mucosal patterns after a short teaching session. Thus, diagnostic skill in magnified endoscopy seems to be easily transposed from examination of the stomach to examination of Barrett's esophagus.

Some of the last generation of European scopes have been equipped to provide both NBI and magnification; for example, 40 times magnification (macroscopy) is possible with Dual Focus (Olympus, Tokyo, Japan). In the near future, more than 100 times magnification (optical zoom) will be provided with the Laser EO series (Fujinon Co., Tokyo, Japan). Singh et al reported the benefits of Dual Focus for the diagnosis of dysplastic Barrett's esophagus, which had $100 \%$ sensitivity and $100 \%$ negative predictive value [12]. Furthermore, $8.1 \%$ of the cases in this study involved LGD, and none of the areas of LGD were mistakenly classified as normal mucosa. This last finding requires further confirmation.

Based on these reports, we should consider the need for magnified endoscopy in Europe, with the provision of organized teaching of the required skills. Learning by studying typical pictures is a first step of the teaching program, but it does not produce the diagnostic ability acquired during real-time endoscopy. In the second step, hands-on training, the skills are acquired that will enable us to generate the same high quality pictures with our endoscopes that our Japanese colleagues produce.

The question we should answer now is whether magnification should be used for detection during the entire Barrett's examination or whether magnification should be used after an abnormality has been detected without magnification, in order to characterize the abnormal area more precisely. In patients with long segments of Barrett's esophagus, however, it seems almost impossible to examine the entire area with magnification, which underscores the need to use NBI without magnification for detection.

To summarize, these various results underscore the need for a precise endoscopic diagnosis, with time and careful attention taken to detect areas of HGD in Barrett's esophagus. NBI examination is the reference for the detection of HGD, and its effectiveness for characterization is probably increased by magnification. With the widespread availability of new endoscopes, we should routinely use magnification to usher in a new era of diagnostic endoscopy. Furthermore, the current results of endoscopic resection for the lesions of Barrett's esophagus are not perfect because of the difficulty encountered in defining margins precisely. NBI magnification can be helpful for the precise identification of dysplastic edges and for effective marking, thus increasing our R0 resection rate [13]. Nevertheless, at present, the results for LGD are not sufficient that the random biopsy protocol can be discontinued. Although the sequence of targeted biopsies followed by random biopsies is time-consuming, it is currently the most effective strategy for detecting HGD lesions without missing undetectable LGD.

\section{Competing interests: None}

\section{References}

1 Singh M, Bansal A, Curvers WL et al. Observer agreement in the assessment of narrowband imaging system surface patterns in Barrett's esophagus: a multicenter study. Endoscopy 2011; 43: 745 - 751

2 Pech 0 , Gossner L, Manner $H$ et al. Prospective evaluation of the macroscopic types and location of early Barrett's neoplasia in 380 lesions. Endoscopy 2007; 39: 588-593

3 Gono K, Obi T, Yamaguchi $M$ et al. Appearance of enhanced tissue features in narrow-band endoscopic imaging. J Biomed Opt 2004; 9: $568-577$

4 Kara MA, Ennahachi M, Fockens $P$ et al. Detection and classification of the mucosal and vascular patterns (mucosal morphology) in Barrett's esophagus by using narrow band imaging. Gastrointest Endosc 2006; 64: $155-166$

5 Wolfsen HC, Crook JE, Krishna M et al. Prospective, controlled tandem endoscopy study of narrow band imaging for dysplasia detection in Barrett's esophagus. Gastroenterology 2008; 135: 24-31

6 Mannath J, Subramanian V, Hawkey CJ et al. Narrow band imaging for characterization of high grade dysplasia and specialized intestinal metaplasia in Barrett's esophagus: a meta-analysis. Endoscopy 2010; 42: $351-359$

7 Sharma P, Hawes RH, Bansal A et al. Standard endoscopy with random biopsies versus narrow band imaging targeted biopsies in Barrett's oesophagus: a prospective, international, randomised controlled trial. Gut 2013; 62: 15-21

8 Anagnostopoulos GK, Yao K, Kaye P et al. Novel endoscopic observation in Barrett's oesophagus using high resolution magnification endoscopy and narrow band imaging. Aliment Pharmacol Ther 2007; 26: $501-507$

9 Sharma P, Marcon N, Wani S et al. Non-biopsy detection of intestinal metaplasia and dysplasia in Barrett's esophagus: a prospective multicenter study. Endoscopy 2006; 38: 1206-1212

10 Baldaque-Silva F, Marques M, Lunet $N$ et al. Endoscopic assessment and grading of Barrett's esophagus using magnification endoscopy and narrow band imaging: impact of structured learning and experience on the accuracy of the Amsterdam classification system. Scand J Gastroenterol 2013; 48: 160-167

11 Singh R, Anagnostopoulos GK, Yao $K$ et al. Narrow-band imaging with magnification in Barrett's esophagus: validation of a simplified grading system of mucosal morphology patterns against histology. Endoscopy 2008; 40: $457-463$

12 Singh $R$, Shahzad MA, Tam $W$ et al. Preliminary feasibility study using a novel narrow-band imaging system with dual focus magnification capability in Barrett's esophagus: is the time ripe to abandon random biopsies? Dig Endosc 2013; 25: 02151 - 156

13 Neuhaus H, Terheggen G, Rutz EM et al. Endoscopic submucosal dissection plus radiofrequency ablation of neoplastic Barrett's esophagus. Endoscopy 2012; 44: 1105-1113 